\title{
Primary research \\ Beat-to-beat changes in stroke volume precede the general
circulatory effects of mechanical ventilation: a case report
}

Nina Nelson* and Birgitta Janerot-Sjöberg ${ }^{\dagger}$

*Department of Health and Environment, Division of Pediatrics, University Hospital, Linköping, Sweden

${ }^{+}$Department of Medicine and Care, Division of Clinical Physiology, University Hospital, Linköping, Sweden

Correspondence: Nina Nelson, MD, PhD, Department of Health and Environment, Division of Pediatrics, Faculty of Health Sciences, University Hospital, SE-581 85 Linköping, Sweden. Tel: +4613 222000; fax: +4613 148265; e-mail: nina.nelson@lio.se

Received: 11 September 2000

Revisions requested: 14 November 2000

Revisions received: 28 November 2000

Accepted: 3 December 2000

Published: 5 January 2001
Critical Care 2001, 5:41-45

(C) 2001 Nelson and Janerot-Sjöberg, licensee BioMed Central Ltd (Print ISSN 1364-8535; Online ISSN 1466-609X)

\begin{abstract}
Background: The haemodynamic as well as the ventilatory consequences of mechanical ventilation can be harmful in critically ill neonates. Newly developed ventilatory lung protective strategies are not always available immediately and in an acute situation the haemodynamic changes caused by mechanical ventilation can affect the oxygen delivery considerably. We report the case of a male neonate who was treated with conventional pressure-controlled mechanical ventilation because of respiratory distress and progressive respiratory acidosis resulting from meconium aspiration. Because of poor arterial oxygenation despite 100\% inspired oxygen and increased ventilator settings, echocardiography was performed to exclude central haemodynamic reasons for low oxygen delivery.
\end{abstract}

Method: Doppler echocardiography was used for the measurement of stroke volume and cardiac output. Pulse oximetry and aortic blood pressure were monitored continuously.

Results: Echocardiography revealed no cardiac malformations or signs of persistent fetal circulation. When inspiratory pressures and duration were increased, beat-to-beat variation in stroke volume preceded decay in cardiac output. Stroke volume variations and oxygen saturation values guided ventilator settings until extracorporal membrane oxygenation could be arranged for. After recovery and discharge 4 weeks later the boy is progressing normally.

Conclusion: Because oxygen delivery is dependent on both blood flow and arterial oxygen content, measurement of cardiac output as well as left heart oxygen saturation is a useful guide to optimizing oxygen delivery. This case report demonstrates how Doppler echocardiographic monitoring of beat-tobeat changes in stroke volume can be used to detect early negative haemodynamic effects of increased mechanical ventilation settings before cardiac output is affected.

Keywords: cardiac output, Doppler echocardiography, haemodynamics, mechanical ventilation, newborn infant

\section{Introduction}

Meconium aspiration syndrome (MAS) is an acute illness affecting full-term babies immediately after birth. Prenatal warning signs, if present, are non-specific and late.
Transportation to special neonatal intensive care units is not always possible. Newly developed ventilatory lung protective strategies such as high-frequency ventilation and/or nitric oxide might not be immediately available. For

$\mathrm{CO}=$ cardiac output $\mathrm{ECMO}=$ extracorporal membrane oxygenation; $\mathrm{LV}=$ left ventricular; $\mathrm{MAS}=$ meconium aspiration syndrome; $\mathrm{PEEP}=$ positive end-expiratory pressure; PIP = peak inspiratory pressure. 
patients with MAS, conventional pressure-controlled mechanical ventilation might therefore be the only option available while waiting for other measures to be taken.

In the acute situation the immediate haemodynamic effect of mechanical ventilation can considerably affect oxygen delivery. This has been evaluated mainly invasively or by a combination of non-invasive and invasive measurements. An increased positive end-expiratory pressure (PEEP) may reduce cardiac output (CO) [1] as a result of decreased preload and increased right ventricular afterload [2]. During positive-pressure lung inflation, the combination of an increased right ventricular afterload and a shift in myocardial compliance, followed by decreased left ventricular (LV) preload, account for the decrease in LV stroke volume $[3,4]$. Especially in neonates the $\mathrm{CO}$ is highly dependent on intrapleural pressure [5]. The left ventricle has a limited ability to change $\mathrm{CO}$ in response to volume loading conditions, and the sympathetic innervation is incomplete or functionally immature in the heart of a newborn infant [6]. However, even when $\mathrm{CO}$ is reduced as a result of high PEEP, pre-term infants can maintain blood pressure by increased afterload [7] and normal blood pressure does not guarantee normal systemic flow [8].

Critically ill neonates often have combined circulatory and respiratory problems, and rapid and repetitive techniques for the bedside assessment of vital functions are essential for effective treatment. Doppler echocardiography is widely used as a non-invasive method for measuring pulmonary and systemic blood flow, including $\mathrm{CO}$. It is accurate especially for detecting changes in $\mathrm{CO}$ and useful even in infants [6]. Variability in stroke volume with preserved $\mathrm{CO}$ during mechanical ventilation has, to our knowledge, not previously been reported. We here present the case of a patient with MAS in which early beat-to-beat-changes in stroke volume, as determined on-line by Doppler echocardiography, guided the ventilator settings to levels not affecting overall central blood flow.

\section{Patient and methods Clinical case}

Signs of fetal asphyxia prompted acute caesarean section at 41 weeks of gestation in a previously healthy primipara. The patient's birth weight was $3610 \mathrm{~g}$ and the Apgar score was 4-8-8. Heavily meconium-stained amniotic fluid was aspirated from the trachea and larynx. A pneumothorax was successfully drained. Progressive acidosis and respiratory difficulties necessitated mechanical ventilation with 100\% oxygen through a pressure-limited time-cycled continuousflow ventilator (Babylog 2; Dräger, Lübeck, Germany). A suitable high-frequency ventilator was not available at the time. In spite of an increasing pressure setting, oxygen saturation deteriorated progressively. Echocardiography showed no signs of organic heart disease or persistent fetal circulation. Dimensional values for both right and left ventricles were in the lower normal range.

\section{Methods}

Blood pressure was monitored with a TmSet1 $(30 \mathrm{ml}$ flush; Codan Triplus AB, Kirchseeon, Germany) attached to an indwelling umbilical arterial argyle catheter (French 5). Oxygen saturation was measured on the right hand by pulse oximetry (OxiNellcor Sensor II N25, Pleasanton, California, USA) and bipolar electrocardiography leads were applied.

Ultrasound Doppler measurements were obtained from the transthoracic five-chamber apical view with a CFM750 echocardiograph $(5 \mathrm{MHz}$ imaging, $4 \mathrm{MHz}$ Doppler; VingMed Sound AB, Horten, Norway). With the patient supine a pulsed-wave Doppler sample volume of $0.4 \mathrm{~cm}^{2}$ was placed centrally in the LV outflow tract proximal to the aortic valve and spectral Doppler recordings were made [9]. The transducer beam was kept as close to parallel to the blood flow as possible; no angle correction was made because the angle was judged to be less than $20^{\circ}$. The flow area, which was assumed to be circular, was calculated from the mean of three two-dimensional diameter measurements of the LV outflow tract in parasternal longaxis view. Beat-to-beat changes in stroke volume (systolic velocity-time integral multiplied by flow area) were calculated and oxygen saturation was measured while ventilator adjustments were made. Changes from baseline values as well as changes within the respiratory cycle were calculated. CO was calculated as the product of heart rate and mean stroke volume.

\section{Results}

Details are given in Table 1 and Fig. 1.

\section{Recordings at baseline}

In spite of increasing ventilator settings and 100\% oxygen, oxygenation was poor. Flow calculation by echocardiography at baseline ventilator settings (see Table 1 for settings and results) showed no increased beat-to-beat variation in electrocardiographic R-R interval (less than 10\%) or stroke volume (less than 4\%, which is the coefficient of variation of systolic velocity-time integral measurements, reported previously from our laboratory [10]) and the mean blood pressure from the monitors remained unchanged (within $\pm 3 \mathrm{mmHg}$ ). Oxygen saturation remained low (46\%) and no pressure plateau was visible on the ventilator airway pressure display at this baseline setting.

\section{Recordings during ventilator manipulation}

Central circulatory effects of peak inspiratory pressure

When peak inspiratory pressure (PIP) was increased further by $2 \mathrm{cmH}_{2} \mathrm{O}$ (to $40 \mathrm{cmH}_{2} \mathrm{O}$ ), a small decrease in LV $\mathrm{CO}$ was recorded. The maximal stroke volume increased slightly but we found a $30 \%$ beat-to-beat variation in stroke volume. This effect was further exaggerated when the inspi- 
Table 1

Ventilatory and haemodynamic effects (lower panel) caused by changes in ventilator settings (upper panel)

\begin{tabular}{|c|c|c|c|c|c|c|c|}
\hline & aseline (Fig. 1a) & I & $\|$ & III & IV (Fig. 1c) & $V($ Fig. 1b) & $\mathrm{VI}$ \\
\hline VR $\left(\min ^{-1}\right)$ & 60 & 60 & 60 & 40 & 60 & 60 & 75 \\
\hline I:E ratio & $1: 2$ & $1: 2$ & $1: 2$ & $1: 2$ & $1: 1$ & $1: 1$ & 1:1 \\
\hline IT (ms) & 330 & 330 & 330 & 500 & 500 & 500 & 400 \\
\hline $\mathrm{PIP}\left(\mathrm{cmH}_{2} \mathrm{O}\right)$ & 38 & 40 & 42 & 40 & 40 & 38 & 38 \\
\hline VPP & No & No & No & Yes & Yes & Yes & No \\
\hline $\mathrm{O}_{2}$ saturation $(\%)$ & 46 & 77 & 79 & 82 & 73 & 85 & 87 \\
\hline $\mathrm{BP}_{\text {mean }}(\mathrm{mmHg})$ & 35 & 34 & 32 & 34 & 36 & 35 & 37 \\
\hline $\mathrm{HR}$ (beats/min) & 107 & 106 & 103 & 106 & 110 & 107 & 103 \\
\hline $\mathrm{SV}_{\max }(\mathrm{ml})$ & 6.2 & 6.6 & 5.6 & 6.4 & 6.6 & 7.9 & 6.2 \\
\hline I:E SV ratio & 1.0 & 0.7 & 0.6 & 0.3 & 0.2 & 0.2 & 0.9 \\
\hline $\mathrm{CO}(\mathrm{ml} / \mathrm{min})$ & 655 & 610 & 445 & 410 & 416 & 635 & 612 \\
\hline $\mathrm{Hb}$ oxygen delivery $(\mathrm{ml} / \mathrm{min})$ & 52 & 80 & 60 & 58 & 52 & 92 & 91 \\
\hline
\end{tabular}

Inspired oxygen fraction $\left(\mathrm{FiO}_{2}\right)$ was 1.0, and PEEP was kept at $5 \mathrm{cmH}_{2} \mathrm{O}$. Baseline ventilatory settings are shown in italics and changes from baseline are shown in bold. VR, ventilatory rate; I, inspiratory; E, expiratory; T, time; VPP, ventilatory pressure plateau; BP, blood pressure; HR, heart rate. For spectral ultrasound Doppler measurements from the left ventricular outflow tract: SV, stroke volume [area under curve during ejection $(\mathrm{cm})$ multiplied by flow area $\left(0.9 \mathrm{~cm}^{2}\right)$ ]; $\mathrm{CO}$, from $\mathrm{SV}_{\text {mean }} \times \mathrm{HR}$ calculated cardiac output; haemoglobin $(\mathrm{Hb})$ oxygen delivery was calculated from haemoglobin content, $\mathrm{O}_{2}$ saturation and $\mathrm{CO}$.

ratory time was increased and also when the ventilatory rate was reduced. A further increase in inspiratory pressure resulted in an overall reduction in Doppler measurements and consequently a decrease in $\mathrm{CO}$.

\section{Central circulatory effects of inspiratory time and}

inspiratory : expiratory ratio

Measurements of maximal blood flow velocity and stroke volume were stable during inspiration and expiration when inspiratory : expiratory ratio increased from $1: 2$ to $1: 1$ if inspiratory time was kept constant (ie an increase in ventilator rate). An increase in inspiratory time to $0.5 \mathrm{~s}$ caused an inspiratory plateau but also an immediate cyclic increase and a decrease in LV stroke volume, initially keeping the CO constant. When PIP was increased, a stroke volume variation remained but the $\mathrm{CO}$ decreased. When inspiratory time was reduced to $0.4 \mathrm{~s}$, respiratory variation in stroke volume was again very small and the $\mathrm{CO}$ returned to the baseline value.

Heart rate, blood pressure and oxygen saturation

The heart rate was $103-110$ beats $/ \mathrm{min}$. The systolic and diastolic aortic blood pressures were $42-46 / 28-29 \mathrm{mmHg}$ (mean $32-37 \mathrm{mmHg}$ ) according to the monitor throughout the procedure. A detailed analysis of beat-to-beat changes in blood pressure, pulse pressure or heart rate within these limits was not performed. Arterial oxygen saturation rose from $46 \%$ to a maximum of $87 \%$ with a slight increased inspiratory time and frequency without seriously affecting stroke volume or CO. Oxygen saturation values above $80 \%$

\section{Figure 1}

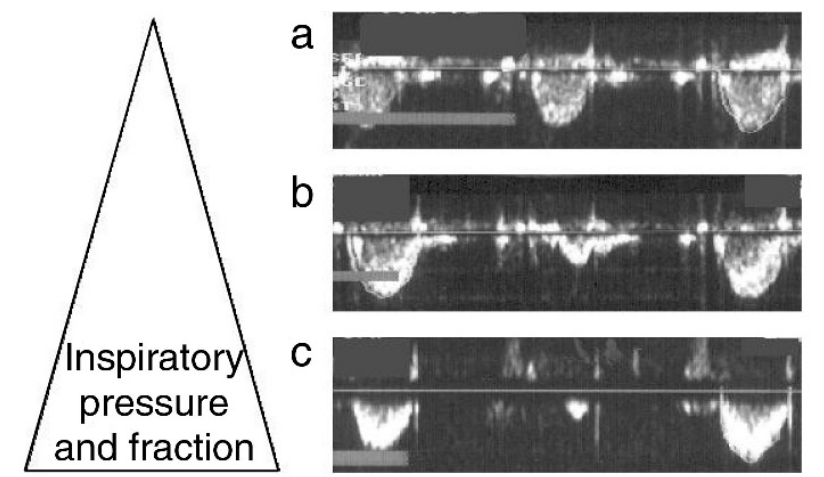

Beat-to-beat changes in stroke volume: ultrasound Doppler records from three consecutive heart beats performed from the apex with the sample volume positioned in the left ventricular outflow tract, (a) at basal ventilator settings; (b) when inspiratory time was prolonged; (c) when inspiratory time as well as PIP was increased. Detailed information on ventilator settings for (a), (b) and (c) is presented in Table 1.

were otherwise recorded independently of the central circulatory effects of ventilation.

\section{Further course}

Neither high-frequency ventilation nor nitric oxide was available for the patient at the time of the study. The baby was not stable enough for transportation to another unit and a national team considered that the baby fulfilled the 
criteria to be accepted for extracorporal membrane oxygenation (ECMO), which took place 10 hours later. In the meantime the arterial blood pressure and heart rate were stable and echocardiography together with oxygen saturation guided the ventilator settings. ECMO was continued for 3 days, mechanical ventilation for a further 2 days and supplementary oxygen for a further week. The baby was initially tube fed and eventually allowed home at the age of 4 weeks. At discharge, computed tomography of the brain was normal, as was his neurological status; the boy is progressing normally.

\section{Discussion}

Lees has reported [6] that the measurement of ventricular output by Doppler echocardiography provides guidance to respirator settings. Our results confirm the poor relation between blood pressure and $\mathrm{CO}$ in neonates [8]. An increased beat-to-beat variation in pulse pressure and a decreased CO induced by PEEP is reported in adults [4]. We show here that in a neonate a decrease in $\mathrm{CO}$ induced by increased inspiratory pressure or time is preceded by beat-to-beat variation in stroke volume. Doppler echocardiography can detect those early changes and in this regard is a useful bedside diagnostic tool in the neonatal intensive care unit.

Most studies on the haemodynamic effects of mechanical ventilation in infants have concerned airway pressure, especially PEEP, and its effect on circulation $[2,4,7]$. It is still uncommon to evaluate the LV circulatory effects of altered ventilator settings. Both inspiratory time and PIP caused serious central circulatory effects in our patient. Cheifetz et al [2] showed, in an animal study, deleterious effects on the circulation by prolongation of the inspiratory time. Our results that showed a reduced LV stroke volume every other heartbeat fitted well with the ventilator rate's being roughly half of the heart rate. Maroto et al [11] noticed, in pre-term infants, increased LV filling during the inspiratory phase of intermittent positive-pressure ventilation. Suggested reasons for an increase in inspiratory LV volume at high intrathoracic pressures include alveolar vessel squeezing, decreased LV compliance, and decreased LV afterload in combination with a phase lag because of a long pulmonary transit time [12]. Michard et al [13] showed nicely the effect of volume expansion and preload on respiratory changes in pulse pressure. The mechanism by which LV function is altered during ventilatory manoeuvres in a given subject remains controversial, as pointed out by Pinsky [12].

Neonatal respiratory diseases often need rapid changes in ventilation. In a recent review on pediatric respiratory failure [14], new lung-protective and oxygenation improving ventilatory modes, and treatment with nitric oxide or surfactant and ECMO are discussed. However, full-term neonates with respiratory problems are born in any hospital, regard- less of the level of the neonatal intensive care unit, and the circulatory effects of conventional ventilator settings have to be considered. As well as the pulmonary effects not discussed here, ventilator settings can cause harmful cardiovascular effects, which reduce the oxygen supply to the tissues despite an increase in arterial oxygen saturation [5]. These acute effects on left heart output were monitored in our patient by Doppler echocardiography.

In a recent study on adults with acute lung injury [4], Michard et al report that a high cyclic variation in pulse pressure during mechanical ventilation without PEEP predicts the CO decrease when PEEP is applied. They also show that a change in pulse pressure is a more reliable indicator of fluid responsiveness than a change in systolic pressure. The beat-to-beat variation in blood pressure was not specifically measured in our patient but the increased beat-to-beat variation in stroke volume occurred before $\mathrm{CO}$ decreased. The role of pulse pressure variation has not to our knowledge been evaluated in neonates in whom the relation between blood pressure and $\mathrm{CO}$ is poor [8].

Our case report confirms that mean blood pressure, heart rate and arterial oxygenation in the clinically available setting might not provide enough information to ensure that the oxygen supply to the tissues is adequate in the management of critically ill babies on mechanical ventilation. Because oxygen delivered to the tissues is dependent on both blood flow and arterial oxygen content, a non-invasive stroke volume measurement provides useful information. An increased inspiratory pressure and duration can initially cause an increased beat-to-beat variation in stroke volume while $\mathrm{CO}$ remains unaffected. Not until LV volume loading and compliance are severely disturbed will the $\mathrm{CO}$ be affected. As well as pulmonary effects themselves, Doppler echocardiographic monitoring of beat-to-beat variation in stroke volume, preceding the decay in $\mathrm{CO}$, might therefore be an important indicator in the guidance of the ventilator settings to prevent further tissue hypoxia.

\section{Acknowledgement}

BJ-S was supported by the Swedish Medical Foundation (grant no. 99P-12313). Support was also obtained from the Swedish Heart Lung Foundation.

\section{References}

1. Jardin F, Farcot JC, Boisante L, Curien N, Margairaz A, Bourdarias JP: Influence of positive end-expiratory pressure on LV performance. N Engl J Med 1981, 304:387-392.

2. Cheifetz IM, Craig DM, Quick G, McGovern JJ, Cannon ML, Ungerleider RM, Smith PK, Meliones JN: Increasing tidal volumes and pulmonary overdistention adversely affect pulmonary vascular mechanics and cardiac output in a pediatric swine model. Crit Care Med 1998, 26:710-716.

3. Jardin F, Delorme G, Hardy A, Auvert B, Beauchet A, Bourdarias JP: Reevaluation of haemodynamic consequences of positive pressure ventilation: emphasis on cyclic right ventricular afterloading by mechanical lung inflation. Anesthesiology 1990, 72:966-970. 
4. Michard F, Chembla D, Richard C, Wysocki M, Pinsky M, Lecarpentier $\mathrm{Y}$, Teboul J-L: Clinical use of respiratory changes in arterial pulse pressure to monitor the hemodynamic effects of PEEP. Am J Resp Crit Care Med 1999, 159:935-939.

5. Gullberg N, Winberg P, Sellden H: Pressure support ventilation increases cardiac output in neonates and infants. Paediatr Anaesth 1996, 6:311-315.

6. Lees $\mathrm{MH}$ : Cardiac output determination in the neonate. $J$ Pediatr 1983, 102:709-711.

7. Hausdorf G, Hellwege $\mathrm{HH}$ : Influence of positive end-expiratory pressure on cardiac performance in premature infants: a Doppler-echocardiographic study. Crit Care Med 1987, 15: 661-664.

8. Kluckow M, Evans N: Relationship between blood pressure and cardiac output in preterm infants requiring mechanical ventilation. J Pediatr 1996, 129:506-512.

9. Labovitz AJ, Buckingham TA, Habermehl K, Nelson J, Kennedy $\mathrm{HL}$, Williams GA: The effects of sampling site on the twodimensional echo-Doppler determination of cardiac output. Am Heart J 1985, 109:327-332.

10. Janerot-Sjöberg B, Wranne B: Cardiac output determined by ultrasound-Doppler: clinical applications. Clin Physiol 1990, 10:463-473.

11. Maroto E, Fouron JC, Teyssier G, Bard H, van Doesburg NH, Cartwright $D$ : Effect of intermittent positive pressure ventilation on diastolic ventricular filling patterns in premature infants. J Am Coll Cardiol 1990, 16:171-174.

12. Pinsky MR: The haemodynamic consequences of mechanical ventilation: an evolving story. Intensive Care Med 1997, 23: 493-503.

13. Michard F, Boussat $S$, Chembla D, Anguel N, Marcat A, Lecarpentier Y, Richard C, Pinsky M, Teboul J-L. Relation between respiratory changes in arterial pulse pressure and fluid responsiveness in septic patients with acute circulatory failure. Am J Resp Crit Care Med 2000, 162:134-138.

14. Doctor A, Arnold J: Mechanical support of acute lung injury: options for strategic ventilation. In New Horizons: The Science and Practice of Acute Medicine. Edited by Goldstein B, Arnold J. Soc Crit Care Med 1999, 7:359-438. 